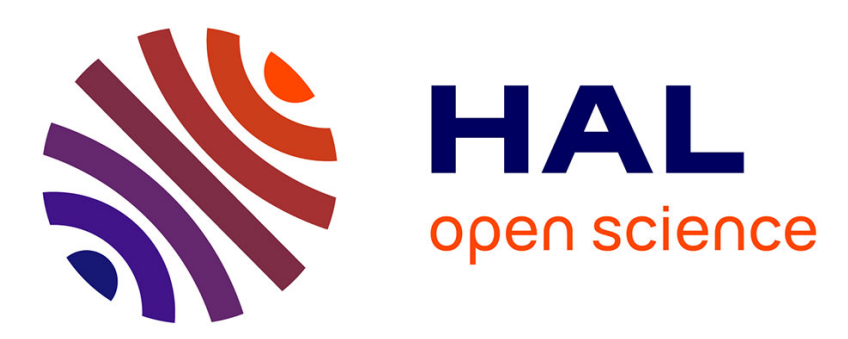

\title{
Adsorption and STM imaging of polycyclic aromatic hydrocarbons on graphene
}

\author{
Yannick J. Dappe, M. Andersen, R. Balog, L. Hornekær, Xavier Bouju
}

\section{To cite this version:}

Yannick J. Dappe, M. Andersen, R. Balog, L. Hornekær, Xavier Bouju. Adsorption and STM imaging of polycyclic aromatic hydrocarbons on graphene. Physical Review B: Condensed Matter and Materials Physics (1998-2015), 2015, 91 (4), 10.1103/PhysRevB.91.045427 . cea-01366506

\section{HAL Id: cea-01366506 https://hal-cea.archives-ouvertes.fr/cea-01366506}

Submitted on 14 Sep 2016

HAL is a multi-disciplinary open access archive for the deposit and dissemination of scientific research documents, whether they are published or not. The documents may come from teaching and research institutions in France or abroad, or from public or private research centers.
L'archive ouverte pluridisciplinaire HAL, est destinée au dépôt et à la diffusion de documents scientifiques de niveau recherche, publiés ou non, émanant des établissements d'enseignement et de recherche français ou étrangers, des laboratoires publics ou privés. 


\title{
Adsorption and STM imaging of polycyclic aromatic hydrocarbons on graphene
}

\author{
Y. J. Dappe \\ Service de Physique de l'Etat Condensé, DSM/IRAMIS/SPEC, CNRS UMR 3680, CEA Saclay, 91191 Gif sur Yvette Cedex, France \\ M. Andersen, R. Balog, ${ }^{\dagger}$ and L. Hornekær \\ Interdisciplinary Nanoscience Center and Department of Physics and Astronomy, Aarhus University, DK-8000 Aarhus C, Denmark \\ X. Bouju \\ Centre d'élaboration de matériaux et d'études structurales (CEMES), Nanosciences Group, UPR CNRS 8011, 29 rue Jeanne-Marvig, \\ POB 94347, F-31055 Toulouse Cedex 4, France
}

(Received 26 August 2014; revised manuscript received 24 December 2014; published 22 January 2015)

\begin{abstract}
The structural characterization of polycyclic aromatic hydrocarbon molecules adsorbed on graphene is of fundamental importance in view of the use of graphene or graphene nanoribbons for electronic applications. Before reaching this point, one has to determine the structure of the adsorbed molecules. Here, we study the case of benzene, coronene, and hexabenzocoronene on a graphene layer. First, the adsorption properties of single molecules are calculated using first-principles calculations at the level of density functional theory. We benefit from a recent scheme, particularly adapted for weakly adsorbed molecules, allowing us to precisely calculate the van der Waals contribution. Then, scanning tunneling microscopy (STM) is used to produce images of self-assembled molecules comparing different theoretical approaches to experimental observations. Finally, we consider the imaging of isolated molecules, and we show how the STM tip influences the molecule position by soft mechanical interaction during the scanning process.
\end{abstract}

DOI: 10.1103/PhysRevB.91.045427

PACS number(s): 68.37.Ef, 68.43.Fg, 71.15.Mb

\section{INTRODUCTION}

Polycyclic aromatic hydrocarbon (PAH) molecules such as benzene, coronene, or hexabenzocoronene (HBC), constitute building blocks for more complex molecules [1,2]. The use of PAHs as molecular skeletons in combination with chemical functionalization leads to molecules of high interest for molecular electronics [3-6], for example. Consequently, the study of these building blocks and their interaction with metallic surfaces or graphitic materials is of fundamental importance. The characterization of their structural and electronic properties is a prerequisite for more complex studies such as electronic transport. In that manner, this work is focused on these two aspects, using density functional theory (DFT) for structural aspects, and scanning tunneling microscopy (STM) image calculation for the electronic characterization.

In addition, since the interaction between these molecules and graphene is dominated by weak covalent and van der Waals interactions $[7,8]$, this constitutes also a model system for the study of dispersion interactions. Even though several approaches have been elaborated recently, these interactions remain complicated to handle, especially at the microscopic level. This is mainly due to the nonlocal character of the noncovalent bonding. For example, some methods have been proposed on the basis of DFT [9-13] with relatively successful results, but are limited by the size of the considered systems and the computational resources, depending on the considered calculation scheme. Additionally, inclusion of many-body contributions improves the accuracy of DFT calculations [14].

\footnotetext{
*yannick.dappe@cea.fr

†Present address: CIC nanoGUNE, Tolosa Hiribidea, 76, E-20018 Donostia-San Sebastian, Spain.
}

On the other hand, semiempirical methods remain important references in the field $[15,16]$, but the parameters still have to be fitted for each new system to be studied. Regarding the specific adsorption of PAH molecules based on the DFT formalism, some studies have been achieved [17-19] on graphene or metallic surfaces, for structural characterization or influence on graphene electronic properties.

Here, our purpose is twofold. Following an intermolecular perturbation theory combined with DFT [20,21], we determine accurately the structural properties of PAH adsorbed on graphene. Second, we calculate STM images of benzene, coronene, and HBC and compare to experimental results. The influence of perturbations from the tip during the scanning process is investigated from a simple force-field parametrization of the van der Waals interactions between molecule and tip.

In Sec. II, we will present our calculation methodology. We will recall our intermolecular perturbation theory plus DFT to determine the structural configuration of the PAH molecules adsorbed on graphene. Then, we will present the adsorption results in Sec. III, and in Sec. IV A, we will show the STM characterization of these systems. Finally, in Sec. IV B we will discuss the tip influence on the molecule during the scanning process.

\section{COMPUTATIONAL METHODS AND EXPERIMENTS}

\section{A. DFT calculations}

In order to perform STM image calculations of PAHs adsorbed on graphene and to get a valid comparison with experiments, we need to accurately characterize the atomic structures of these systems. Therefore we have used DFT calculations to determine the equilibrium distances of the 
different PAHs with respect to graphene. Since the PAHgraphene interaction is dominated by $\pi$ stacking and van der Waals (vdW) interactions, we have used the previously developed LCAO- $S^{2}+$ vdW formalism [20,21]. This approach is based on DFT in combination with an intermolecular perturbation theory to describe weak covalent and $\mathrm{vdW}$ interactions. Each interacting subsystem, namely in this case the PAH (benzene, coronene, or HBC) and the graphene plane, are calculated separately by DFT. The DFT computational scheme as well as the theoretical foundations underlying our calculations - a very efficient DFT localized orbital molecular dynamics technique (FIREBALL) - have been described in full details elsewhere [22-25].

We first analyze all the systems involved in this study by using a self-consistent version of the Harris-Foulkes LDA functional [26,27]—instead of the traditional Kohn-Sham (KS) functional based on the electronic density-where the KS potential is calculated by approximating the total charge by a superposition of spherical charges around each atom. The FIREBALL simulation package uses a localized optimized minimal basis set [28], and the self-consistency is achieved over the occupation numbers through the Harris functional [29]. Besides, the LDA exchange-correlation energy is calculated using the efficient multicenter weighted exchange-correlation density approximation (McWEDA) $[23,24]$. To these DFT calculations we add "weak interactions," which can be seen as two opposite contributions. The first one, named "weak chemical" interaction, is due to the small overlaps between electronic densities of the interacting subsystems. Therefore, this energy can be determined as an expansion of the wave functions and operators with respect to these overlaps. Typically, in graphitic or hydrocarbonated materials this contribution is repulsive [21].

The second contribution is the pure van der Waals interaction, which finds its origin in charge fluctuations in each subsystem, arising from oscillating dipoles whose interaction gives the attractive part of the cohesive energy. This interaction is treated in the dipolar approximation and added in perturbations to the total energy of the system.

The balance of the two contributions gives the equilibrium configuration of the system. This formalism [20,21] has already provided excellent results in the study of graphenegraphene or molecule-graphene interactions [30] or encapsulated molecules in nanotubes [31].

\section{B. STM image calculations}

For the monolayer of coronene molecules on graphene we compared two approaches to simulated STM images, namely DFT calculations coupled to the Tersoff-Hamann approximation [32] (DFT-TH) and the elastic scattering quantum chemistry (ESQC) method [33,34]. Whereas ab initio DFT calculations could potentially give a more accurate description of the electronic structure of the system [35-39] compared to the semiempirical extended Hückel approximation implemented in ESQC, STM simulations in the Tersoff-Hamann approximation are rather crude since the STM tip is merely modeled as a sphere with a single $s$-wave state. The advantage of the ESQC method lies in allowing for the simultaneous description of the surface, the adsorbates in the tunneling junction, the tip apex, and the tip bulk as well as the explicit treatment of the tunneling current. Even though DFT-TH provides good simulated images of bare layered substrates such as graphite and graphene [35,40-42], we will show in the following that its accuracy is inferior to ESQC for coronene on graphene. We have therefore preferred the ESQC method for all subsequent STM calculations of PAHs on graphene. The here reported STM simulations of monolayer coronene on graphene in the Tersoff-Hamann approximation are based on previous DFT calculations [43]. In the Tersoff-Hamann approximation the local density of states (LDOS) is integrated from the Fermi level to the bias voltage. Different tunneling currents can be modeled by considering different isocontour surfaces for the LDOS. In the ESQC method, the tunneling current is evaluated at low bias voltage from the full scattering matrix of the tunneling junction. The system is described by a monoelectronic Hamiltonian, where the matrix elements are evaluated within the semiempirical extended Hückel approximation. The valence orbitals of the atoms are described by a Slater-type basis set. Using standard Slater parameters for carbon, we found that the current perpendicular to the graphene layers of the graphite bulk is rather small [44]. To keep the electronic properties intact and to mimic as close as possible the experimental system, we therefore considered a decoupled graphene layer deposited on a $\mathrm{Cu}(111)$ substrate. It is well known that on the copper substrate the graphene layer is weakly bonded and preserves its gapless structure $[45,46]$. Moreover, as we will show, there is an excellent agreement with the experimental STM images of PAHs on graphite. Indeed, a graphene layer may adopt a particular structure once adsorbed, especially on a rough $\mathrm{SiO}_{2}$ substrate, on $\mathrm{SiC}[47,48]$, on metallic surfaces [46,49] such as $\mathrm{Ru}(0001)$, $\mathrm{Rh}(111), \mathrm{Pt}(111), \operatorname{Ir}(111)$, or Ni(111), or on h-BN [50]. Even if a moiré pattern was observed on $\mathrm{Cu}(111)$ with STM [51], as well as preferential sticking of molecules on some parts of the moiré [52], one anticipates that the reconstruction of the graphene layer is small enough to consider an unperturbed substrate for molecular adsorption. For the $\mathrm{Cu}$ surface and the tip we limited the basis set to a single $4 s$ orbital, except for the apex $\mathrm{Cu}$ atom which was modeled with two Slater exponents to allow for long-range interactions with the adsorbates in the tunneling junction. The carbon and hydrogen atoms in the tunneling junction were described by full standard $2 s 2 p$ and $1 s$ basis sets, respectively. Such a parametrization has been already used with success $[53,54]$. Notice that the tip apex is ended by a single atom supported by a perfect trigonal aggregate (its structure is threefold). The probe structure does not influence the image of molecular adsorbate because the tip-sample distance is such that the second plane of the tip aggregate does not contribute directly to the main tunneling current. The control of the tip apex structure and its orientation with respect to the surface is much less sensitive in STM than in the noncontact atomic force microscopy (AFM) [55-58], due to the $z$ dependence of the tunnel current variation which is more acute for STM than for AFM.

\section{Experimental setup}

Measurements were performed using the home-built Aarhus STM [59] under ultrahigh vacuum (UHV) at a base 
pressure of $5 \times 10^{-10}$ mbar. The grade 1 HOPG substrate was obtained from SPI Supplies. Coronene samples of $99 \%$ purity were provided by Sigma-Aldrich. In order to remove the contamination in coronene samples introduced by handling and transfer procedures, the glass crucible filled with coronene was heated in vacuum to $120^{\circ} \mathrm{C}$ for 2 hours. The ordered monolayer of coronene was then achieved by heating the crucible up to $180{ }^{\circ} \mathrm{C}$ for $120 \mathrm{~s}$ while keeping it $5 \mathrm{~cm}$ away from HOPG substrate. During deposition, the substrate was kept at room temperature but afterwards slowly (ramp $1 \mathrm{~K} / \mathrm{s}$ ) heated to $100^{\circ} \mathrm{C}$ to desorb any coronene multilayers. This approach yields a densely packed and highly stable monolayer of coronene, which allows for performing STM measurements even at room temperature. However, in order to obtain the highest possible stability and resolution, all presented STM images are obtained while keeping the substrate at temperatures of about 140-150 K.

\section{ADSORPTION}

In this section, we present the main results of our structural calculations for the case of benzene, coronene, and $\mathrm{HBC}$ adsorption on graphene. The metallic substrate was not considered as it brings only a van der Waals background energy to the PAH molecule. All these calculations have been performed using the LCAO- $S^{2}+v d W$ as described previously. In each case, we have calculated the adsorption potential energy of each molecule as a function of the distance to the graphene plane for several $(x, y)$ positions of the molecules in the plane, and we have kept the optimal energy for each $(x, y)$ position in order to build the potential energy surface (PES). In Fig. 1, we present those PESs for the adsorption of benzene, coronene, and $\mathrm{HBC}$ on graphene, respectively. As a main result, we can observe that the three PESs share the same geometric characteristics; i.e., the most stable structure is obtained for an AB-like stacking, similarly to the interaction between two graphene planes. Hence, we obtain a minimum in the potential energy when the center of the molecule coincides with an atom of the graphene plane. Our calculations also indicate that the average distance of these graphitic molecules to the graphene plane is naturally close to the graphene-graphene distance (3.35 $\AA$ experimentally, 3.1 $\AA$ in the LCAO- $S^{2}+$ $\mathrm{vdW}$ formalism). More precisely, this distance lies between 2.9 and $3.0 \AA$ for benzene on graphene and 3.0 and $3.1 \AA$ for coronene on graphene, which means that the effect of corrugation is really small on the molecule-graphene distance. However the energy variation is noticeable, between 0.54 and $0.62 \mathrm{eV}$, and 1.70 and $2.05 \mathrm{eV}$ per benzene and coronene molecules, respectively. Regarding the $\mathrm{HBC}$ molecule, the distance range is more important, between 2.9 and $3.1 \AA$. This behavior can be explained by the bigger size of the molecule, which results in a larger sensitivity towards the graphene corrugation. The adsorption energy lies between 2.9 and $3.5 \mathrm{eV}$ per molecule. In that case, one can also stress that the molecule-graphene distance is closer to the graphene-graphene distance, as another consequence of the bigger size of HBC. Finally, if we analyze the adsorption energy values with respect to the number of carbon atoms in the molecule, we obtain the following. For benzene, the energy per carbon atom is around $90-103 \mathrm{meV}$. This value should be compared to our previously published value for the graphenegraphene interaction (between 60-72 meV/atom [20]), which was obtained using the same methodology. While the value for the graphene-graphene interaction is in good agreement with results obtained using the $\mathrm{vdW}(\mathrm{TS})$ functional [12] and the optB88-vdW functional [60], our value for benzene is significantly higher. This can be explained by the lack of quadrupole-quadrupole interactions in our model (which remains at the dipole-dipole interaction level), which is important for small molecules, and decreases with the size of the molecule for symmetry reasons, like in the graphene-graphene configuration, for example. In the coronene case, the energy range is around $71-85 \mathrm{meV} /$ atom, in good agreement with quantum chemical calculations at the PM6-DH2 level [61], and DFT calculations using the optB88-vdW functional [60]. As another comparison, the energy value found in this case using the vdW-DF functional gives a slightly lower result, around $63 \mathrm{meV} /$ atom [60]. However this method is known to give results at the lower boundary of the energy range. Finally, the $\mathrm{HBC}$ adsorption is characterized by an energy range of about $69-83 \mathrm{meV} /$ atom. As stated previously, the increasing size of the molecules stabilizes the adsorption energy per atom until reaching the value for the graphene-graphene interaction.

In the next section, we will use the above determined structures to model STM experiments on the PAH coronene adsorbed on graphite. Notice that adding more graphene layers to describe the graphite substrate does not modify the present
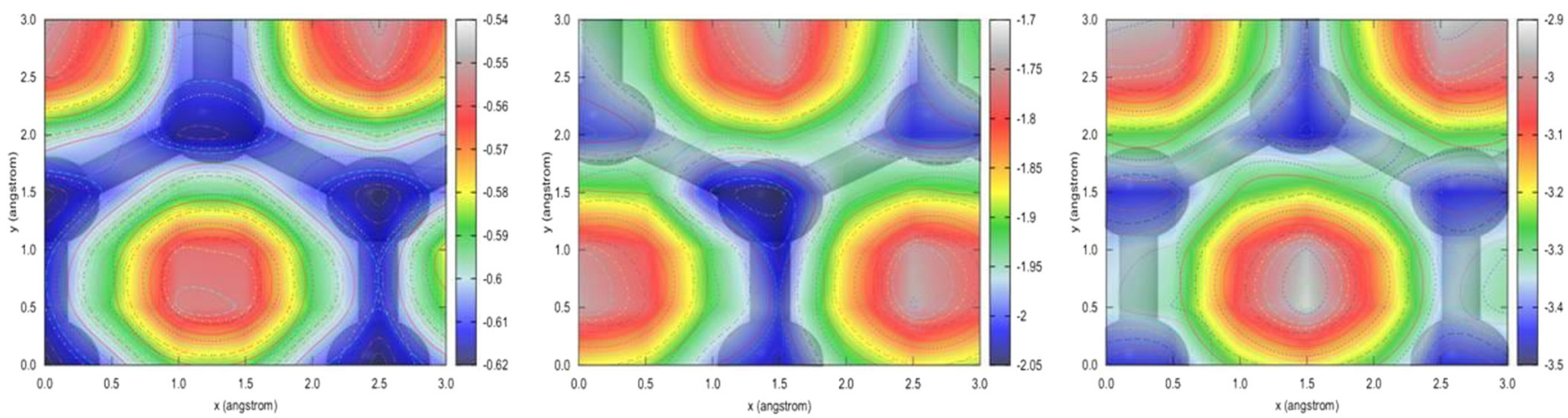

FIG. 1. (Color online) Contour energy plot of the adsorption potential energy on graphene, calculated using the LCAO- $S^{2}+\mathrm{vdW}$ formalism for (left panel) benzene, (middle panel) coronene, and (right panel) HBC. The $(x, y)$ position refers to the center of the molecule. The color scale refers to potential energy in $\mathrm{eV}$. The graphene lattice is overlaid in gray. 
results. Indeed, considering a graphite substrate just adds a background of van der Waals energy due to the large interlayer distance and does not modify significantly the corrugation energy of the PES.

\section{STM CHARACTERIZATION OF PAH ADSORPTION}

\section{A. STM images: Theory-experiments comparison}

After determining the adsorption geometry of the molecules, we performed STM calculations of PAH molecules adsorbed on a supported graphene layer. In order to check the accuracy of the method, we first consider a monolayer of coronene molecules on graphene. In this case, the adsorption structure has been previously determined from DFT, employing the vdW-optB88 functional [43], and from a LEED study [62]. In Fig. 2 we compare experimental STM images [(a)-(c)] extracted from large images showing extensive selfassembled monolayer, ESQC-STM calculated images [(d)-(f)] and DFT-TH [(g)-(i)] simulated STM images of the coronene monolayer obtained at energies ranging from around $3 \mathrm{eV}$ below the Fermi level of the substrate to around $4 \mathrm{eV}$ above the Fermi level. The three selected bias voltages in the experimental images are representative for the different imaging modes observed when varying the voltage within the range accessible in STM experiments. For the calculated images many different bias voltages and currents were tested. Using the ESQC method, we were able to reproduce qualitatively very well the experimental images with current and bias conditions close to experimental ones. Here, one has to notice the change of the molecule images with a contrast reversal according to the bias voltage. For bias around $-3.0 \mathrm{~V}[(\mathrm{a})$ and (d)], each coronene molecule looks like a donut with a circular protrusion for the peripheral rings of the coronene, and a small depression corresponding to the central ring. An opposite situation occurs for bias voltage around 4.2 eV [(c) and (f)], where the molecule appears as a dark spot and the substrate between the molecules is revealed by a bright and large signature, exhibiting a sixfold symmetry. Now, between these two ranges at $V_{t}=1.74 \mathrm{~V}$, we experimentally observe that this last signature shows a triangular shape with three distinct bumps. We recover such a behavior in the calculated image with a higher voltage, i.e., at $\sim 3.5 \mathrm{~V}$. This discrepancy should be explained by the different substrates considered, but a qualitative agreement is revealed. Apart from this, the concordance between experimental and calculated images is very good. Based on calculated spectra, the change of image appearance is related to the particular molecular resonances probe by the STM bias voltage.
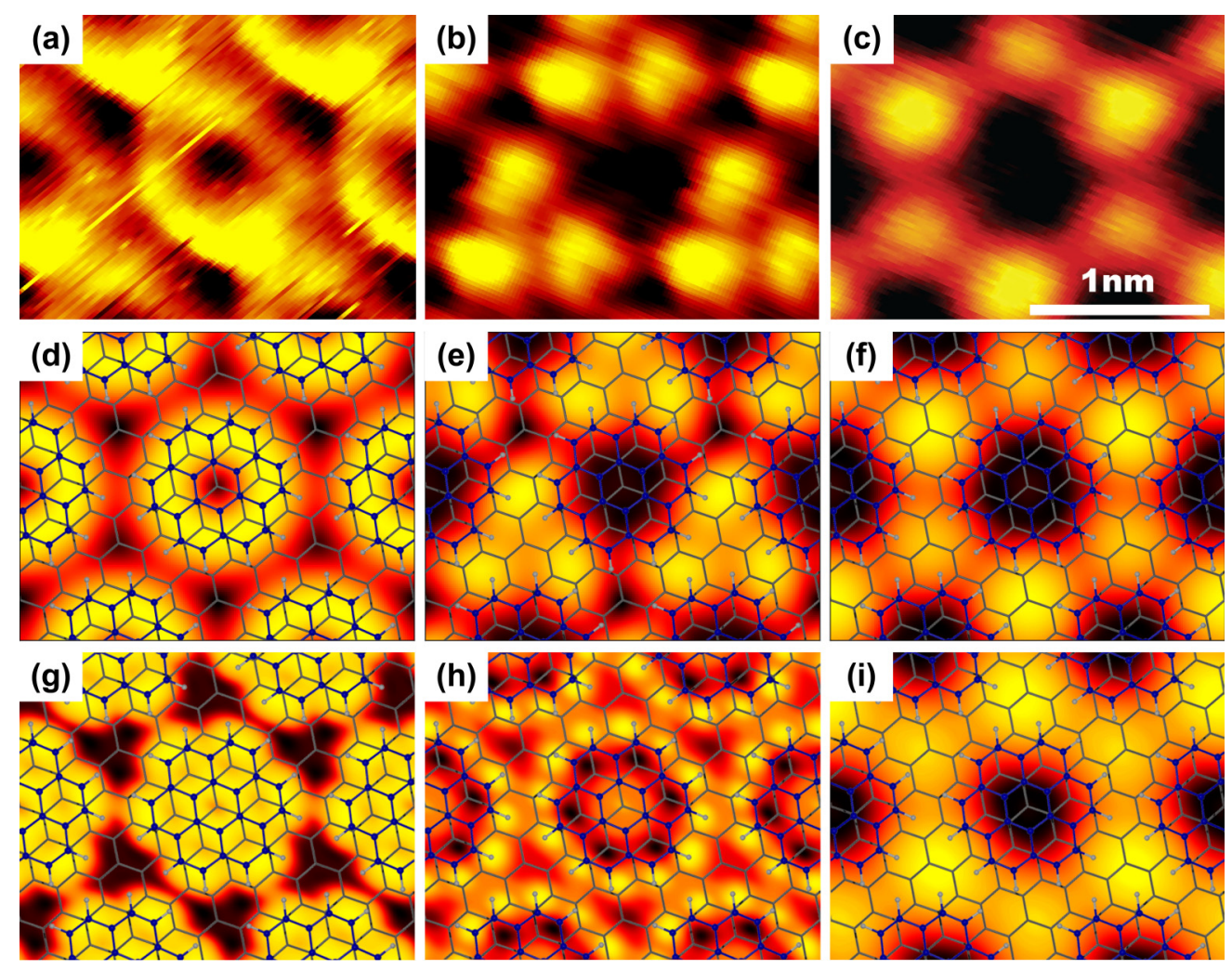

FIG. 2. (Color online) (a)-(c) Experimental STM images of a monolayer of coronene on graphite. Tunneling conditions are (a) $V_{t}=$ $-3.11 \mathrm{~V}, I_{t}=70 \mathrm{pA}$, (b) $V_{t}=1.74 \mathrm{~V}, I_{t}=120 \mathrm{pA}$, and (c) $V_{t}=4.14 \mathrm{~V}, I_{t}=110 \mathrm{pA}$. (d)-(f) ESQC calculated constant-current STM images of a monolayer of coronene adsorbed on graphene with the superimposed atomic structure. The graphene lattice is depicted in gray whereas the coronene is depicted with carbon atoms in dark blue and hydrogen atoms in white. Tunneling conditions and color scales (black to yellow) are (d) $V_{t}=-2.83 \mathrm{~V}, I_{t}=70 \mathrm{pA},[5.75 \AA, 6.92 \AA]$, (e) $V_{t}=3.46 \mathrm{~V}, I_{t}=23 \mathrm{pA},[6.73 \AA, 7.91 \AA]$, (f) $V_{t}=4.20 \mathrm{~V}, I_{t}=2.2 \mathrm{pA},[5.11 \AA$, $6.36 \AA$ ㄱ. (g)-(i) DFT-TH simulated STM images. Tunneling conditions are (g) $V_{t}=-2.83 \mathrm{~V}, I_{t}=500 \mathrm{pA}$, (h) $V_{t}=3.46 \mathrm{~V}, I_{t}=500 \mathrm{pA}$, (i) $V_{t}=4.20 \mathrm{~V}, I_{t}=500 \mathrm{pA}$. 

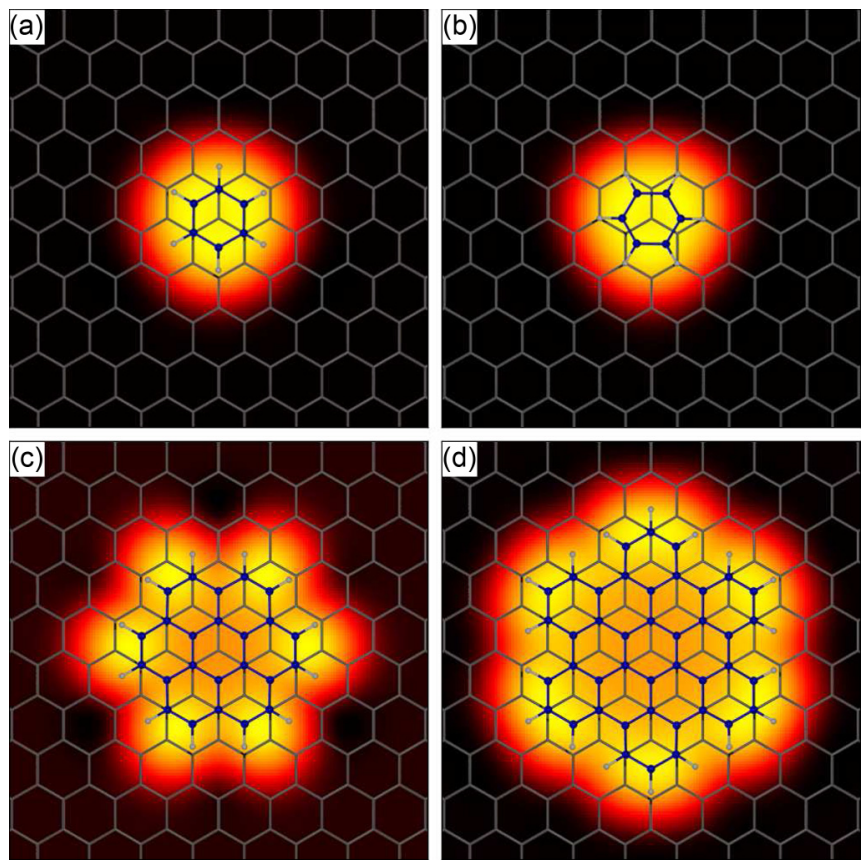

FIG. 3. (Color online) Constant-current calculated STM images of (a) benzene in stacked configuration, (b) benzene rotated $30^{\circ}$, (c) coronene in stacked configuration, and (d) hexabenzocoronene in stacked configuration. The coloring of the superimposed atomic structures is the same as in Fig. 2. Tunneling conditions and color scales (black to yellow) are $V_{t}=-0.1 \mathrm{~V}, I_{t}=1 \mathrm{pA}$, [5.25 ̊, $\left.6.30 \AA\right]$ for all images.

The STM images simulated from DFT-TH show varying degrees of agreement with the measured images. At high bias voltage the agreement is quite good, and at low bias voltage there is a similarity, but the simulated image lacks the depression in the center of the molecule. However, for the medium bias voltage-in spite of testing many different bias voltages and LDOS isocontour values-the triangular shape observed in the experimental images and in the ESQC calculated images could not be observed. As mentioned previously, the Tersoff-Hamann approximation is rather crude, and the observed discrepancies could be related to the failure to take into account a realistic tip and the explicit treatment of all tunneling paths in the system, all of which is accounted for in the ESQC method. The good performance of ESQC allows us to have confidence in this method for the study of isolated molecules as described next.

In Fig. 3 we show ESQC calculated STM images of the isolated benzene, coronene, and HBC molecules obtained just below the Fermi level within the HOMO-LUMO gap. Since for benzene, the stacked [Fig. 3(a)] and the $30^{\circ}$ rotated [Fig. 3(b)] configurations are very close in adsorption energy we consider the two situations. At this energy the calculated images of both configurations are circular features almost identical in appearance. For coronene [Fig. 3(c)] and HBC [Fig. 3(d)] the calculated images show a sixfold symmetry with six bright protrusions on the edge of the molecule and a darker contrast towards the center. For all molecules, the calculated images at this energy are nearly independent of the orientation of the molecule with respect to the graphene layer (only shown for the two benzene configurations). At these imaging conditions, it is not possible to resolve the inner structure of the molecule; only an apparent shape can be obtained. In consequence, the determination of the precise position of the molecule with respect to the substrate is difficult without the help of calculations.

In Fig. 4, we show calculated images at $2.4 \mathrm{eV}$ above the Fermi level for the benzene molecule in (a) the stacked configuration, (b) the $30^{\circ}$ rotated configuration, and (c) displaced away from the optimal adsorption site. At this energy there is a pronounced influence of the rotation or translation of the molecule with respect to the substrate.

As a final remark on those calculations, we want to stress here that the considered imaging conditions (low current and high tip-sample distance) generate low electric fields, which allows us to neglect shifts of the molecular levels in the calculations.

\section{B. Influence of the STM tip: Molecular manipulation}

As shown in a previous paper [43], the diffusion energy of each molecule on the graphene substrate is relatively low, about tens of meV (see in Fig. 1). This is the reason why it is not trivial to image single molecules without disturbing them during the scanning process. One has to use low tunneling currents to
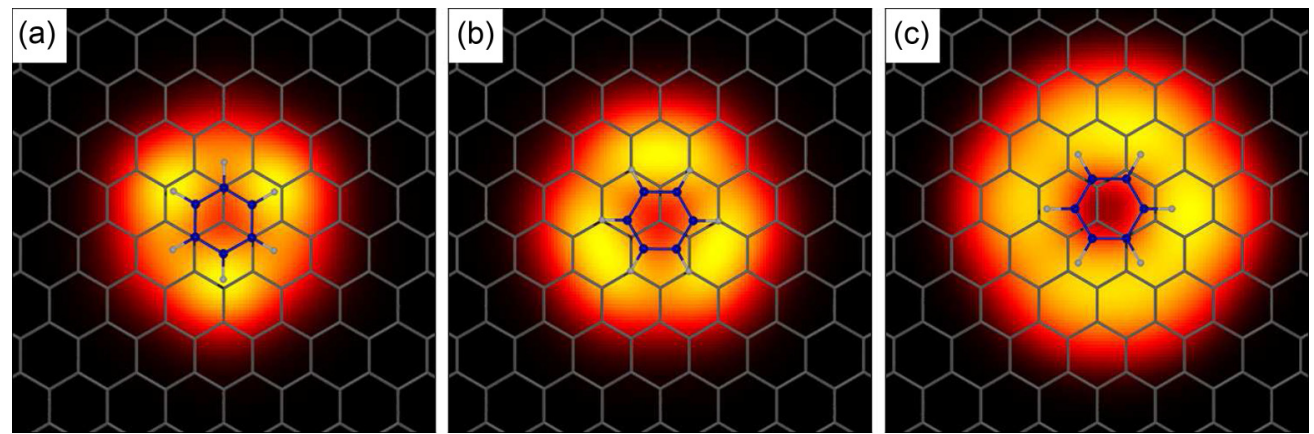

FIG. 4. (Color online) Constant-current calculated STM images of (a) benzene in stacked configuration, (b) benzene rotated $30^{\circ}$, and (c) benzene displaced away from the optimal adsorption site. The coloring of the superimposed atomic structures is the same as in Fig. 2. Tunneling conditions are $V_{t}=2.4 \mathrm{~V}, I_{t}=1 \mathrm{pA}$ for all images. Color scales (black to yellow) are (a) [5.15 $\left.\mathrm{A}, 5.89 \AA\right]$, (b) [5.15 $\left.\mathrm{A}, 5.97 \AA\right]$, and (c) [5.15 ̊, $7.02 \AA]$. 
reach high enough tip-sample distance to not displace the adsorbate. We have checked this property by calculating STM images of a single HBC molecule in the constant-height mode: At a fixed tip height $\left(z_{\text {tip }}\right)$ and for each $(x, y)$ position of the probe during the scanning, we have calculated the total potential energy of the $\mathrm{HBC}$ molecule, that is to say the tipmolecule energy $E_{\mathrm{MT}}+$ the molecule-substrate energy $E_{\mathrm{MS}}$. The $E_{\mathrm{MT}}$ energy is evaluated by a standard MM4 force-field parametrization for van der Waals interactions [63-65]:

$$
E_{\mathrm{MT}}=\sum E_{a b}\left(1.84 \times 10^{5} e^{-12 / p}-2.25 \times p^{6}\right),
$$

where $p=r_{a b} / r$, with $a$ and $b$ standing for $\mathrm{Cu}, \mathrm{C}$, or $\mathrm{H}$ atoms. The summation runs over all the atoms of the molecule and of the metallic tip separated by the interatomic distance $r$. The mixed parameters follow the MM4 rules, $E_{a b}=\sqrt{E_{a} E_{b}}$ and $r_{a b}=r_{a}+r_{b}$. The following MM4 parameters have been used: $E_{\mathrm{Cu}}=12.83, E_{\mathrm{C}}=24.72, E_{\mathrm{H}}=7.37$ in $\mathrm{meV}$, and $r_{\mathrm{Cu}}=2.26, r_{\mathrm{C}}=1.96, r_{\mathrm{H}}=1.64$ in $\AA$. Notice that the considered tip has a [111] trigonal structure with $106 \mathrm{Cu}$ atoms. Once the optimal total energy is found, the molecule is kept at the optimum $(X, Y, Z)$ position and the tunneling current is then calculated. The images in Fig. 5 are obtained by scanning the surface line by line starting from the bottom left to the top (rapid scanning direction along the $y$ axis), and the reverse after a tip displacement increment of $\Delta x=0.8 \AA$
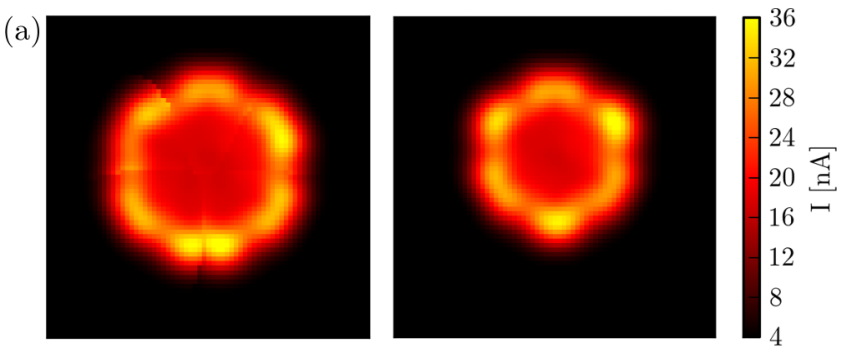

(b)
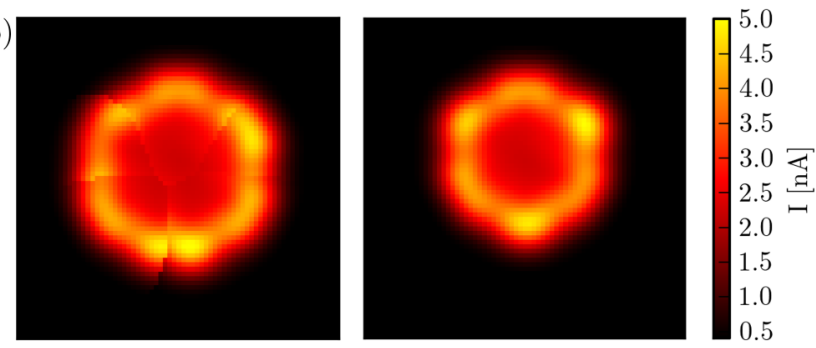

(c)
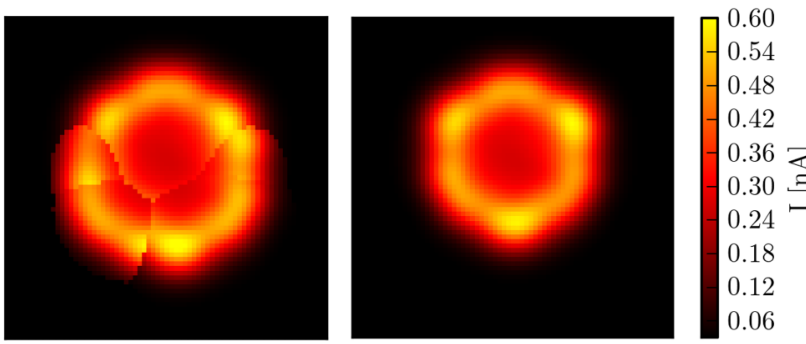

FIG. 5. (Color online) Comparison between the STM imaging of hexabenzocoronene in constant-height mode at (a) $9 \AA$, (b) $10 \AA$, and (c) $11 \AA$ when the molecule is allowed to move under influence of the tip (left panels) and when the molecule is kept fixed (right images). The images are obtained at the Fermi energy. (the slow scanning direction), until the top right point is reached. One can observe that the molecule is gently attracted and repelled by the tip around its central position. This induces an increase of the apparent shape of the imaged molecule if one compares with the case where the molecule is not free to move (right panels in Fig. 5). This increase reaches at least $\sim 31 \%$ of the apparent area. More interestingly, the shape corresponding to the molecule is largely distorted and it becomes difficult to recover the actual and fine structure of the molecule when the tip is close to the surface. Actually, the tip acts as a van der Waals trap whose depth is modulated by the tip height $[66,67]$. For lower tip-sample distances, a mechanical action occurs on the molecule with manipulation possibility during the scanning [68-70]. Even for $z_{\text {tip }}=9 \AA$, i.e., for a tip-molecule distance greater than $6 \AA$ in our case (taking into account the molecule-graphene distance), the molecule is sensitive to the interaction of the tip. This constitutes an important piece of information in the perspective of using single molecules to functionalize graphene layers or graphene nanoribbons. Indeed, their observation can be altered by the tip-molecule interaction, leading to incorrect interpretations of the experimental results. Nevertheless, it remains that local-probe based methods, such as STM and atomic force microscopy (AFM), are the most adapted techniques to characterize the structure of adsorbates [71], even for those with low adsorption and diffusion energies. On the other hand, this property can also be turned into an advantage, as manipulating molecules opens important potentialities for designing new electronic devices. Here, we have presented a specific case of weakly interacting molecules on graphene, as an academic case of study for molecular manipulation.

\section{CONCLUSION}

In this work, we have investigated the interaction of PAH molecules with a graphitic substrate. Considering weak covalent and van der Waals interactions, we have determined the energetic landscape of benzene, coronene, and HBC adsorbed on graphene. We have observed that the graphene corrugation influences the adsorption energy very little, except for the HBC molecule due to its bigger size. Also, the molecular adsorption energy per atom tends to the expected graphene-graphene interaction energy per atom when the size of the PAH molecule is increasing, which is in good agreement with previous studies.

For the monolayer of coronene on graphene we have compared STM images calculated from ESQC and DFT-TH to experimental images. Whereas the ESQC method showed a very good agreement with the experiments, we could not recover all experimentally observed features in the images with the DFT-TH method. This could be caused by the very approximate treatment of the tunneling process in the latter method. For isolated PAHs, STM calculations at energies around specific molecular levels exhibited a significant influence of the molecular displacement with respect to the substrate, whereas nothing was observed for energies in the gap. This can be attributed to the symmetry of the molecular levels in interaction with the electronic states of the 
graphene plane. Finally, since an isolated molecule presents a high mobility with respect to a molecular self-assembly, we have modeled the influence of the STM tip on the molecular position during the scanning process. In particular we have shown a nonnegligible displacement, even at high tip height, leading to a deformation of the STM image. Nevertheless, this property can also be exploited for molecular manipulation to design electronic devices at a fundamental level. Consequently, PAHs on graphene constitute an academic case study of weakly interacting systems for molecular manipulation.

\section{ACKNOWLEDGMENT}

Part of this work was performed using high-performance computing resources from the Calcul en Midi-Pyrénées (CALMIP) facilities (Grant No. 2011-[P0832]).
[1] D. Peña, in Ideas in Chemistry and Molecular Sciences: Advances in Synthetic Chemistry, Chap. 11, edited by B. Pignataro (Wiley-VCH, Weinheim, 2010).

[2] J. M. Alonso, A. E. Díaz-Álvarez, A. Criado, D. Pérez, D. Peña, and E. Guitián, Angew. Chem. Int. Ed. 51, 173 (2012).

[3] V. Georgakilas, M. Otyepka, A. B. Bourlinos, V. Chandra, N. Kim, K. C. Kemp, P. Hobza, R. Zboril, and K. S. Kim, Chem. Rev. 112, 6156 (2012).

[4] A. Schlierf, P. Samorì, and V. Palermo, J. Mater. Chem. C 2, 3129 (2014).

[5] S. Wu, M. T. González, R. Huber, S. Grunder, M. Mayor, C. Schönenberger, and M. Calame, Nat. Nanotechnol. 3, 569 (2008).

[6] F. Chen and N. J. Tao, Acc. Chem. Res. 42, 429 (2009).

[7] F. London, Z. Phys. Chem. B11, 222 (1930).

[8] F. London, Z. Phys. 63, 245 (1930).

[9] J. G. Ángyán, I. C. Gerber, A. Savin, and J. Toulouse, Phys. Rev. A 72, 012510 (2005).

[10] D. C. Langreth, M. Dion, H. Rydberg, E. Schröder, P. Hyldgaard, and B. I. Lundqvist, Int. J. Quantum Chem. 101, 599 (2005).

[11] S. Grimme, J. Antony, T. Schwabe, and C. Mück-Lichtenfeld, Org. Biomol. Chem. 5, 741 (2007).

[12] A. Tkatchenko and M. Scheffler, Phys. Rev. Lett. 102, 073005 (2009).

[13] S. Lebegue, J. Harl, T. Gould, J. G. Ángyán, G. Kresse, and J. F. Dobson, Phys. Rev. Lett. 105, 196401 (2010).

[14] R. A. DiStasio, Jr., V. V. Gobre, and A. Tkatchenko, J. Phys.: Condens. Matter 26, 213202 (2014).

[15] L. A. Girifalco and R. A. Lad, J. Chem. Phys. 25, 693 (1956).

[16] P. A. Gravil, M. Devel, Ph. Lambin, X. Bouju, Ch. Girard, and A. A. Lucas, Phys. Rev. B 53, 1622 (1996).

[17] S. D. Chakarova-Käck, E. Schröder, B. I. Lundqvist, and D. C. Langreth, Phys. Rev. Lett. 96, 146107 (2006).

[18] Y.-H. Zhang, K.-G. Zhou, K.-F. Xie, J. Zeng, H.-L. Zhang, and Y. Peng, Nanotechnology 21, 065201 (2010).

[19] M. Wiener, N. S. Rodríguez Lastra, J. Ziroff, F. Forster, P. Puschnig, L. Dösse, K. Müllen, A. Schöll, and F. Reinert, New J. Phys. 14, 113008 (2012).

[20] Y. J. Dappe, M. A. Basanta, F. Flores, and J. Ortega, Phys. Rev. B 74, 205434 (2006).

[21] Y. J. Dappe, J. Ortega, and F. Flores, Phys. Rev. B 79, 165409 (2009).

[22] J. P. Lewis, K. R. Glaesemann, G. A. Voth, J. Fritsch, A. A. Demkov, J. Ortega, and O. F. Sankey, Phys. Rev. B 64, 195103 (2001).

[23] P. Jelínek, H. Wang, J. P. Lewis, O. F. Sankey, and J. Ortega, Phys. Rev. B 71, 235101 (2005).
[24] O. F. Sankey and D. J. Niklewski, Phys. Rev. B 40, 3979 (1989).

[25] J. P. Lewis, P. Jelínek, J. Ortega, A. A. Demkov, D. G. Trabada, B. Haycock, H. Wang, G. Adams, J. Tomfohr, E. Abad, H. Wang, and D. A. Drabold, Phys. Status Solidi B 248, 1989 (2011).

[26] J. Harris, Phys. Rev. B 31, 1770 (1985).

[27] W. M. C. Foulkes and R. Haydock, Phys. Rev. B 39, 12520 (1989).

[28] M. A. Basanta, Y. J. Dappe, P. Jelínek, and J. Ortega, Comput. Mater. Sci. 39, 759 (2007).

[29] A. A. Demkov, J. Ortega, O. F. Sankey, and M. P. Grumbach, Phys. Rev. B 52, 1618 (1995).

[30] M. Svec, P. Merino, Y. J. Dappe, C. González, E. Abad, P. Jelínek, and J. A. Martin-Gago, Phys. Rev. B 86, 121407(R) (2012).

[31] Y. J. Dappe and J. I. Martinez, Carbon 54, 113 (2013).

[32] J. Tersoff and D. R. Hamann, Phys. Rev. B 31, 805 (1985).

[33] P. Sautet and C. Joachim, Phys. Rev. B 38, 12238 (1988).

[34] P. Sautet and C. Joachim, Chem. Phys. Lett. 185, 23 (1991).

[35] D. Tománek and S. G. Louie, Phys. Rev. B 37, 8327 (1988).

[36] S. Corbel, J. Cerdá, and P. Sautet, Phys. Rev. B 60, 1989 (1999).

[37] W. A. Hofer, A. S. Foster, and A. L. Shluger, Rev. Mod. Phys. 75, 1287 (2003).

[38] J. C. Chen, Introduction to Scanning Tunneling Microscopy (Oxford University Press, Oxford, 2008).

[39] B. A. Janta-Polczynski, J. I. Cerdá, G. Éthier-Majcher, K. Piyakis, and A. Rochefort, J. Appl. Phys. 104, 023702 (2008).

[40] D. Tománek, S. G. Louie, H. J. Mamin, D. W. Abraham, R. E. Thomson, E. Ganz, and J. Clarke, Phys. Rev. B 35, 7790 (1987).

[41] P. Xu, Y. Yang, S. D. Barber, M. L. Ackerman, J. K. Schoelz, I. A. Kornev, S. Barraza-Lopez, L. Bellaiche, and P. M. Thibado, Phys. Rev. B 84, 161409(R) (2011).

[42] P. Xu, Y. Yang, D. Qi, S. D. Barber, M. L. Ackerman, J. K. Schoelz, T. B. Bothwell, S. Barraza-Lopez, L. Bellaiche, and P. M. Thibado, Appl. Phys. Lett. 100, 201601 (2012).

[43] J. D. Thrower, E. E. Friis, A. L. Skov, L. Nilsson, M. Andersen, L. Ferrighi, B. Jørgensen, S. Baouche, R. Balog, B. Hammer, and L. Hornæker, J. Phys. Chem. C 117, 13520 (2013).

[44] P. Sautet and C. Joachim, Ultramicroscopy 42-44, 115 (1992).

[45] P. A. Khomyakov, G. Giovannetti, P. C. Rusu, G. Brocks, J. van den Brink, and P. J. Kelly, Phys. Rev. B 79, 195425 (2009).

[46] E. Voloshina and Y. Dedkov, Phys. Chem. Chem. Phys. 14, 13502 (2012).

[47] P. Mallet, F. Varchon, C. Naud, L. Magaud, C. Berger, and J.-Y. Veuillen, Phys. Rev. B 76, 041403(R) (2007).

[48] F. Castanié, L. Nony, S. Gauthier, and X. Bouju, Beilstein J. Nanotechnol. 3, 301 (2012). 
[49] E. N. Voloshina, E. Fertitta, A. Garhofer, F. Mittendorfer, M. Fonin, A. Thissen, and Y. S. Dedkov, Sci. Rep. 3, 1072 (2013).

[50] M. M. van Wijk, A. Schuring, M. I. Katsnelson, and A. Fasolino, Phys. Rev. Lett. 113, 135504 (2014).

[51] T. Niu, M. Zhou, J. Zhang, Y. Feng, and W. Chen, J. Am. Chem. Soc. 135, 8409 (2013).

[52] J. M. MacLeod and F. Rosei, Small 10, 1038 (2014).

[53] T. Zambelli, P. Jiang, J. Lagoute, S. E. Grillo, S. Gauthier, A. Gourdon, and C. Joachim, Phys. Rev. B 66, 075410 (2002).

[54] M. Alemani, L. Gross, F. Moresco, K.-H. Rieder, C. Wang, X. Bouju, A. Gourdon, and C. Joachim, Chem. Phys. Lett. 402, 180 (2005).

[55] M. P. Boneschanscher, J. van der Lit, Z. Sun, I. Swart, P. Liljeroth, and D. Vanmaekelbergh, ACS Nano 6, 10216 (2012).

[56] P. Pou, S. A. Ghasemi, P. Jelinek, T. Lenosky, S. Goedecker, and R. Perez, Nanotechnology 20, 264015 (2009).

[57] F. Castanié, L. Nony, S. Gauthier, and X. Bouju, J. Phys. Chem. C 117, 10492 (2013).

[58] H. Sang, S. P. Jarvis, Z. Zhou, P. Sharp, P. Moriarty, J. Wang, Y. Wang, and L. Kantorovich, Sci. Rep. 4, 6678 (2014).

[59] E. Lægsgaard, F. Besenbacher, K. Mortensen, and I. Stensgaard, J. Microsc.-Oxford 152, 663 (1988).
[60] J. Björk, F. Hanke, C.-A. Palma, P. Samori, M. Cecchini, and M. Persson, J. Phys. Chem. Lett. 1, 3407 (2010).

[61] E. G. Gordeev, M. V. Polynski, and V. P. Ananikov, Phys. Chem. Chem. Phys. 15, 18815 (2013).

[62] U. Zimmermann and N. Karl, Surf. Sci. 268, 296 (1992).

[63] N. L. Allinger, K. S. Chen, and J. H. Lii, J. Comput. Chem. 17, 642 (1996).

[64] N. L. Allinger, J. Comput. Aided Mol. Des. 25, 295 (2011).

[65] M. Yu, W. Xu, Y. Benjalal, R. Barattin, E. Lægsgaard, I. Stensgaard, M. Hliwa, X. Bouju, A. Gourdon, C. Joachim, T. R. Linderoth, and F. Besenbacher, Nano Res. 2, 254 (2009).

[66] X. Bouju, C. Girard, H. Tang, C. Joachim, and L. Pizzagalli, Phys. Rev. B 55, 16498 (1997).

[67] L. N. Kantorovich and T. Trevethan, Phys. Rev. Lett. 93, 236102 (2004).

[68] J. T. Li, R. Berndt, and W.-D. Schneider, Phys. Rev. Lett. 76, 1888 (1996).

[69] X. Bouju, C. Joachim, and C. Girard, Phys. Rev. B 59, R7845 (1999).

[70] X. Bouju, C. Joachim, C. Girard, and H. Tang, Phys. Rev. B 63, 085415 (2001).

[71] L. Gross, F. Mohn, N. Moll, B. Schuler, A. Criado, E. Guitián, D. Peña, A. Gourdon, and G. Meyer, Science 337, 1326 (2012). 